Vietnam Journal of Mechanics, VAST, Vol.38, No. 4 (2016), pp. 279-293

DOI:10.15625/0866-7136/7118

\title{
FREE VIBRATION OF A CRACKED DOUBLE-BEAM CARRYING A CONCENTRATED MASS
}

\author{
Nguyen Viet Khoa*, Nguyen Van Quang \\ Institute of Mechanics, Vietnam Academy of Science and Technology, Hanoi, Vietnam \\ *E-mail: nvkhoa@imech.ac.vn \\ Received September 21, 2015
}

\begin{abstract}
This paper presents the free vibration of a cracked double-beam carrying a concentrated mass located at an arbitrary position. The double-beam consisting of two different simply supported beams connected by an elastic medium is modelled by using finite element method. The influence of the concentrated mass on the frequencies and mode shapes is investigated. The relationship between the natural frequency and the location of concentrated mass is established and related to the mode shapes. The numerical simulations show that when there is a crack, the frequency of the double-beam changes sharply when the concentrated mass is located close to the crack position. This sharp change can be amplified by wavelet transform and this is useful for crack detection. The crack location can be determined by the location of peaks in the wavelet transform of the relationship between frequency and mass location.
\end{abstract}

Keywords: Natural frequency, mode shape, concentrated mass, crack, double-beam.

\section{INTRODUCTION}

The elastically connected beam structures are widely used in various engineering fields, such as multiple-walled carbon nanotubes, tall building, continuous dynamic vibration absorber, ect. as presented in [1,2]. These complex continuous systems have three important structural performances: weight reduction, strength and stiffness increase, and vibration absorption which can be applied in practice. The vibration analysis of elastically connected double beams has been investigated by many researchers and obtained interesting achievement. Oniszczuk [1] presented the exact solutions for free vibrations of two parallel simply supported beams continuously joined by a Winkler elastic layer. Mao [2] proposed the Adomian modified decomposition method to analyze the free vibration of elastically multiple-beams. Oniszczuk [3] presented undamped forced transverse vibrations of an elastically connected simply supported double-beam system subjected to arbitrarily distributed continuous loads using the modal expansion

(c) 2016 Vietnam Academy of Science and Technology 
method. Rao [4] studied the free response of Timoshenko beam systems taken into account the effects of rotary inertia and shear deformation. Chen and Sheu [5] modelled a composite material by elastically connected beams to analyze the vibration of an axially loaded double Timoshenko beam. Shamalta and Matrikine [6] investigated the steadystate dynamic response of an embedded railway track subjected to a moving train. $\mathrm{Vu}$ et al. [7] proposed an exact method for analyzing the vibration of a double-beam system subjected to a harmonic excitation. Hoppmann [8] proposed a method for solving the differential equations of motion of an elastically connected double-beam system subjected to an impulsive load.

However, many aspects of the vibration problem of double-beam systems such as the influence of concentrated masses, the influence of cracks on the vibration of doublebeam systems have not been addressed, while these influences on the vibration of single beams have been investigated by many researchers. Salarieha and Ghorashi [9] investigated free vibration of Timoshenko beam with finite mass rigid tip load and flexuraltorsional coupling. Liu and Yeh [10] presented Rayleigh-Ritz method in conjunction with beam functions satisfying all end conditions to study the free vibration of a restrained non-uniform beam with intermediate masses. Wu and Lin [11] used an analytical and numerical-combined method to study the free vibration of a uniform cantilever beam with point masses. The influence of concentrated mass on the natural frequencies of a single cantilever beam was investigated. Banerjee [12] proposed a dynamic stiffness method to study the free vibration of beams carrying spring-mass systems. Liu et al. [13] analysed the eigenvalues and eigenfunctions for a beam hinged at both ends by rotational springs and carrying arbitrary located concentrated masses using Laplace transformation method. Goel [14] studied the free vibration of a cantilever beam carrying a concentrated mass at an arbitrary intermediate location.

The influence of the crack(s) on characteristics of single beams such as natural frequencies and mode shapes have also been studied and used for detecting the existence, location and sizes of the crack(s). Gudmundson [15] presented a perturbation method and a transfer matrix approach to investigate the influence of small cracks on the natural frequencies of slender structures. Pandey et al. [16] investigated the change in curvature mode shapes to inspect the location of the crack. Vakil-Baghmisheh [17] applied genetic algorithms for crack detection of beam-like structures. Orhan [18] used finite element method to investigate the relationship between natural frequency of a cracked beam to the depth and location of the crack. Lee et al. [19] investigated the influence of a crack on natural frequencies and mode shapes of a beam. Khaji et al. [20] presented closedform solutions for crack detection problem of Timoshenko beams with various boundary conditions. Caddemi and Calio [21] derived the exact closed-form solution for the vibration modes of the Euler-Bernoulli beam with multiple open cracks. Vakil-Baghmisheh et al. Zhong and Oyadiji [22] presented a wavelet-based method for crack detection of simply-supported beams by continuous wavelet transform of reconstructed modal data. Recently, the author of this paper [23] applied 3D finite elements to calculate the mode shapes of a cracked beam. This study showed that the distortion in mode shapes using 3D element model can be applied for detection small cracks. 
This work aims to investigate the influence of the concentrated mass on the free vibration of a cracked double-beam and its application for crack detection. The natural frequencies and mode shapes of the cracked double-beam carrying a concentrated mass are calculated by using finite element method. When the concentrated mass is attached on the double-beams there are changes in natural frequencies depending on the mass position. The changes in natural frequencies can be related to the amplitudes of the corresponding mode shapes at the position of the concentrated mass. The relationship between the natural frequencies and the mass location is established and called "Mass Location - Frequency" (MLF). When the concentrated mass is located close to crack positions there are sharp changes in the MLFs at these positions. Inspecting these irregular changes by applying wavelet transform, the crack locations can be determined.

\section{FREE VIBRATION EQUATION OF A BEAM WITH CONCENTRATED MASS}

\subsection{Intact double-beam}

The finite element model of the double-beam system consisting of two different Euler-Bernoulli beams with rectangular sections connected by a Winkler elastic layer with stiffness modulus $k_{m}$ per unit length is presented in Fig. 1 . The length of the double-beam is $L$. Each of the main and auxiliary beams is divided by $Q$ equal elements with length of $l$. The main beam carries a concentrated mass $m$ at section $x_{m}$. In this study, the undamped vibrations of the system are considered.

The free motion equation of an element of the double-beam system can be derived by using Hamilton's principle as follows [24].

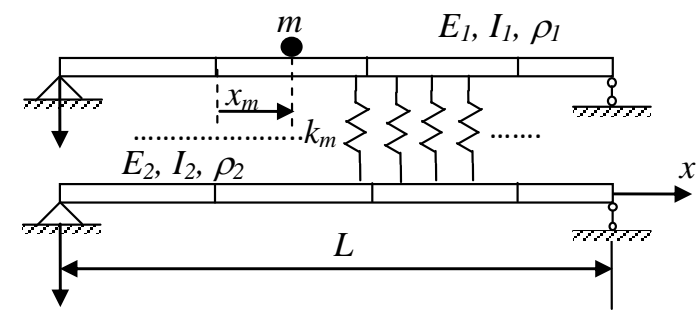

Fig. 1. A double-beam element carrying a concentrated mass

Using a local coordinate system having its origin at the center of the element, and the element is defined from $-l / 2$ to $+l / 2$, the kinetic energy of a double element carrying the concentrated mass can be written as

$$
T=\frac{1}{2} \dot{\mathbf{d}}_{e 1}^{\mathrm{T}}\left(\int_{-l / 2}^{l / 2}\left[m \delta\left(x-x_{m}\right)+\rho_{1}\right] \mathbf{N}^{\mathrm{T}} \mathbf{N} d x\right) \dot{\mathbf{d}}_{e 1}+\frac{1}{2} \dot{\mathbf{d}}_{e 2}^{\mathrm{T}}\left(\int_{-l / 2}^{l / 2} \rho_{2} \mathbf{N}^{\mathrm{T}} \mathbf{N} d x\right) \dot{\mathbf{d}}_{e 2},
$$

where $\rho_{1}$ and $\rho_{2}$ are the material densities of the main and auxiliary elements per unit length, respectively; $\delta$ is delta Dirac function; $\dot{\mathbf{d}}_{e 1}$ and $\dot{\mathbf{d}}_{e 2}$ are the velocity vectors of the main and auxiliary elements; $\mathbf{N}$ is shape function. 
Denote that

$$
\mathbf{m}^{*}=\int_{-l / 2}^{l / 2} m \delta\left(x-x_{m}\right) \mathbf{N}^{\mathrm{T}} \mathbf{N} d x=m \mathbf{N}^{\mathrm{T}} \mathbf{N}, \mathbf{m}_{e 1}=\int_{-l / 2}^{l / 2} \rho_{1} \mathbf{N}^{\mathrm{T}} \mathbf{N} d x, \mathbf{m}_{e 2}=\int_{-l / 2}^{l / 2} \rho_{2} \mathbf{N}^{\mathrm{T}} \mathbf{N} d x,
$$

Substitute (2) into (1) we have

$$
T=\frac{1}{2} \dot{\mathbf{d}}_{e 1}^{T} \mathbf{m}^{*} \dot{\mathbf{d}}_{e 1}+\frac{1}{2} \dot{\mathbf{d}}_{e 1}^{T} \mathbf{m}_{e 1} \dot{\mathbf{d}}_{e 1}+\frac{1}{2} \dot{\mathbf{d}}_{e 2}^{T} \mathbf{m}_{e 2} \dot{\mathbf{d}}_{e 2} .
$$

Here: $\mathbf{m}_{e 1}$ and $\mathbf{m}_{e 2}$ are the element mass matrices of the main and auxiliary beams; $\mathbf{m}^{*}$ is the additional matrix of the concentrated mass.

Denote

$$
\mathbf{m}_{e 1}^{*}=\mathbf{m}_{e 1}+\mathbf{m}^{*}
$$

Substitute (4) into (3), we have

$$
T=\frac{1}{2} \dot{\mathbf{d}}_{e 1}^{T} \mathbf{m}_{e 1}^{*} \dot{\mathbf{d}}_{e 1}+\frac{1}{2} \dot{\mathbf{d}}_{e 2}^{T} \mathbf{m}_{e 2} \dot{\mathbf{d}}_{e 2} .
$$

The potential energy of the system can be obtained

$$
\Pi=\frac{1}{2} \mathbf{d}_{e 1}^{T} \mathbf{k}_{e 1} \mathbf{d}_{e 1}+\frac{1}{2} \mathbf{d}_{e 2}^{T} \mathbf{k}_{e 2} \mathbf{d}_{e 2}+\frac{1}{2}\left(\mathbf{d}_{e 1}^{T}-\mathbf{d}_{e 2}^{T}\right) \mathbf{k}_{m}^{*}\left(\mathbf{d}_{e 1}-\mathbf{d}_{e 2}\right),
$$

where $\mathbf{k}_{e 1}$ and $\mathbf{k}_{e 2}$ are element stiffness matrices of the main and auxiliary beam; $\mathbf{d}_{e 1}$ and $\mathbf{d}_{e 2}$ are the displacement vectors of the main and auxiliary elements, and

$$
\mathbf{k}_{m}^{*}=\int_{-l / 2}^{l / 2} k_{m}(x) \mathbf{N}^{T} \mathbf{N} d x
$$

The Lagrangian can be established

$$
L=T-\Pi
$$

or

$$
\begin{aligned}
L= & \frac{1}{2} \dot{\mathbf{d}}_{e 1}^{T} \mathbf{m}_{e 1} \dot{\mathbf{d}}_{e 1}+\frac{1}{2} \mathbf{d}_{e 1}^{T} \mathbf{m}^{*} \dot{\mathbf{d}}_{e 1}+\frac{1}{2} \dot{\mathbf{d}}_{e 2}^{T} \mathbf{m}_{e 2} \dot{\mathbf{d}}_{e 2}-\frac{1}{2} \mathbf{d}_{e 1}^{T} \mathbf{k}_{e 1} \mathbf{d}_{e 1} \\
& -\frac{1}{2} \mathbf{d}_{e 2}^{T} \mathbf{k}_{e 2} \mathbf{d}_{e 2}-\frac{1}{2}\left(\mathbf{d}_{e 1}^{T}-\mathbf{d}_{e 2}^{T}\right) \mathbf{k}_{m}^{*}\left(\mathbf{d}_{e 1}-\mathbf{d}_{e 2}\right) .
\end{aligned}
$$

Applying Hamilton's principle

$$
\delta \int_{t_{1}}^{t_{2}} L d t=0
$$

with the initial conditions $\delta \mathbf{d}_{e 1}=0$ and $\delta \mathbf{d}_{e 2}=0$ at moments $t=t_{1}$ and $t=t_{2}$, the governing equations for free vibration of an element can be obtained as follows

$$
\begin{aligned}
& \mathbf{m}_{e 1}^{*} \ddot{\mathbf{d}}_{e 1}+\mathbf{k}_{e 1} \mathbf{d}_{e 1}+\mathbf{k}_{m}^{*}\left(\mathbf{d}_{e 1}-\mathbf{d}_{e 2}\right)=\mathbf{0}, \\
& \mathbf{m}_{e 2} \ddot{\mathbf{d}}_{e 2}+\mathbf{k}_{e 2} \mathbf{d}_{e 2}-\mathbf{k}_{m}^{*}\left(\mathbf{d}_{e 1}-\mathbf{d}_{e 2}\right)=\mathbf{0} .
\end{aligned}
$$


Here, $\ddot{\mathbf{d}}_{e 1}$ and $\ddot{\mathbf{d}}_{e 2}$ are the acceleration vectors of the main and auxiliary elements; $\mathbf{0}$ is the zero column vector consisting of four elements.

Finally, the governing equations of free vibration of the double-beam in the global coordinate system can be written as

$$
\begin{aligned}
& \mathbf{M}_{1}^{*} \ddot{\mathbf{D}}_{1}+\mathbf{K}_{1} \mathbf{D}_{1}+\mathbf{K}_{m}^{*}\left(\mathbf{D}_{1}-\mathbf{D}_{2}\right)=\mathbf{0}, \\
& \mathbf{M}_{2} \ddot{\mathbf{D}}_{2}+\mathbf{K}_{2} \mathbf{D}_{2}-\mathbf{K}_{m}^{*}\left(\mathbf{D}_{1}-\mathbf{D}_{2}\right)=\mathbf{0} .
\end{aligned}
$$

Here $\mathbf{M}_{1}^{*}, \mathbf{M}_{2}, \mathbf{K}_{1}, \mathbf{K}_{2}$ are global structural mass and stiffness matrices of the main and auxiliary beams, respectively; $\mathbf{K}_{m}^{*}$ is the global stiffness matrix of the elastic medium; $\mathbf{D}_{1}$ and $\mathbf{D}_{2}$ are column vectors which denote the nodal displacements of the main and auxiliary beams, respectively; $\mathbf{0}$ is the zero column vector assembled from column vectors 0.

Eq. (12) can be rewritten as follows

$$
\mathbf{M D}+\mathbf{K D}=\tilde{\mathbf{0}} .
$$

Here

$$
\begin{aligned}
& \mathbf{M}=\left[\begin{array}{l}
\mathbf{M}_{1}^{*} \\
\mathbf{M}_{2}
\end{array}\right], \quad \mathbf{K}=\left[\begin{array}{cc}
\mathbf{K}_{1}+\mathbf{K}_{m}^{*} & -\mathbf{K}_{m}^{*} \\
-\mathbf{K}_{m}^{*} & \mathbf{K}_{2}+\mathbf{K}_{m}^{*}
\end{array}\right], \\
& \mathbf{D}=\left[\begin{array}{l}
\mathbf{D}_{1} \\
\mathbf{D}_{2}
\end{array}\right], \quad \ddot{\mathbf{D}}=\left[\begin{array}{c}
\ddot{\mathbf{D}}_{1} \\
\ddot{\mathbf{D}}_{2}
\end{array}\right], \quad \tilde{\mathbf{0}}=\left[\begin{array}{l}
\mathbf{0} \\
\mathbf{0}
\end{array}\right] .
\end{aligned}
$$

\subsection{Cracked double-beam}

Fig. 2 shows the cracked double element model. It is assumed that the crack appears on the main beam and the crack only affects the stiffness of main beam but it does not affect the stiffness of auxiliary beam. Meanwhile, the masses of main and auxiliary beams remain constant. A brief description for deriving the element stiffness matrix of a cracked element of the main beam is presented here, more details can be found from the previous papers [25-27].

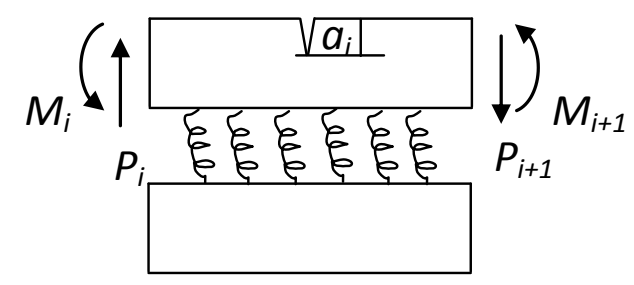

Fig. 2. Model of a cracked double element

The stiffness matrix of the cracked element is derived by applying the principle of virtual work

where

$$
\mathbf{k}_{c}=\mathbf{T}^{T} \tilde{\mathbf{C}} \mathbf{T},
$$

$$
\mathbf{T}=\left[\begin{array}{cccc}
-1 & -l & 1 & 0 \\
0 & -1 & 0 & 1
\end{array}\right]^{T}
$$


$\tilde{\mathrm{C}}$ is flexibility matrix of the cracked element which is the sum of the flexibility matrix of the intact element $\mathrm{C}^{0}$ and the additional flexibility matrix $\mathrm{C}^{1}$ caused by the crack. The generic components of the flexibility matrices $\mathbf{C}^{0}$ and $\mathbf{C}^{1}$ can be calculated from the fracture mechanics as follows

$$
\begin{gathered}
c_{i j}^{(0)}=\frac{\partial^{2} W^{(0)}}{\partial P_{i} \partial P_{j}}, \quad i, j=1,2, \quad P_{1}=P, \quad P_{1}=M, \\
c_{i j}^{(1)}=\frac{\partial^{2} W^{(1)}}{\partial P_{i} \partial P_{j}}, \quad i, j=1,2, \quad P_{1}=P, \quad P_{1}=M,
\end{gathered}
$$

where $W^{(0)}$ is the strain energy of the intact element; $W^{(1)}$ is the additional energy due to the crack; $P$ and $M$ are the shear and bending internal forces at the right node of the element (Fig. 2). Considering the bending only, $W^{(0)}$ and $W^{(1)}$ are obtained as

$$
\begin{aligned}
& W^{(0)}=\frac{1}{2 E I}\left(M^{2} l+M P l^{2}+\frac{P^{2} L^{3}}{3}\right), \\
& W^{(1)}=b \int_{0}^{a}\left(\frac{\left(K_{I M}+K_{I P}\right)^{2}+K_{I I P}^{2}}{E^{\prime}}\right) d a,
\end{aligned}
$$

where

$$
\begin{aligned}
& K_{I M}=\frac{6 M \sqrt{\pi a} F_{I}(s)}{b h^{2}}, \quad K_{I P}=\frac{3 P l \sqrt{\pi a} F_{I}(s)}{b h^{2}}, \quad K_{I I P}=\frac{P \sqrt{\pi a} F_{I I}(s)}{b h}, \\
& F_{I}(s)=\sqrt{\frac{2}{\pi s} \operatorname{tg}\left(\frac{\pi s}{2}\right)} \frac{0.923+0.199\left[1-\sin \left(\frac{\pi s}{2}\right)\right]^{4}}{\cos \left(\frac{\pi s}{2}\right)}, \\
& F_{I I}(s)=\left(3 s-2 s^{2}\right) \frac{1.122-0.561 s+0.085 s^{2}+0.18 s^{3}}{\sqrt{1-s}} .
\end{aligned}
$$

Here, $a$ is the crack depth; $h$ is the thickness; $b$ is the width of the beam; $s=a / h$.

The stiffness matrix of a cracked beam will be assembled from the stiffness matrix of cracked element and intact elements of the corresponding beam. In this study, the cracks are in the main beam, thus the global stiffness matrix $\mathbf{K}_{1}$ of the cracked beam is assembled from the stiffness matrix of the cracked elements derived from (15) and the stiffness matrix of intact elements. Global stiffness matrices $\mathbf{K}_{1}$ and $\mathbf{K}_{2}$ are assembled following Eq. (14) to form the global stiffness matrix $\mathbf{K}$ of the cracked double-beam. Substituting this global matrix K into Eq. (13) and solving this eigenvalue equation, the frequencies and mode shapes of the cracked double-beam carrying a concentrated mass will be obtained.

\section{NUMERICAL SIMULATION}

\subsection{Reliability of the theory}

In order to check the reliability of the theory, a double simply supported beam with parameters adopted from Ref. [1,2] is considered as follows. 
$E_{2} I_{2}=4 \times 10^{6} \mathrm{~N} / \mathrm{m}^{2}, E_{1} I_{1}=2 \times E_{2} I_{2}, \rho_{2} A_{2}=100 \mathrm{~kg} / \mathrm{m}, \rho_{1} A_{1}=2 \times \rho_{2} A_{2}$, $k_{m}=1 \times 10^{5} \mathrm{~N} / \mathrm{m}^{2}, L=10 \mathrm{~m}$.

Table 1. Natural frequencies of a double-beam

\begin{tabular}{|c|c|c|c|}
\hline Natural frequencies (rad/s) & Ref. [1] & Ref. [2] & Present paper \\
\hline $\bar{\omega}_{1}$ & 19.7 & 19.7392 & 19.7392 \\
\hline $\bar{\omega}_{2}$ & 43.5 & 43.4699 & 43.4699 \\
\hline $\bar{\omega}_{3}$ & 79.0 & 78.9568 & 78.9568 \\
\hline $\bar{\omega}_{4}$ & 87.9 & 87.9442 & 87.9442 \\
\hline $\bar{\omega}_{5}$ & 177.7 & 177.6529 & 177.6529 \\
\hline $\bar{\omega}_{6}$ & 181.8 & 181.8256 & 181.8256 \\
\hline
\end{tabular}

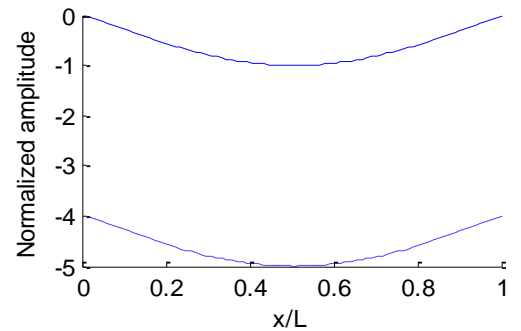

(a) Mode 1

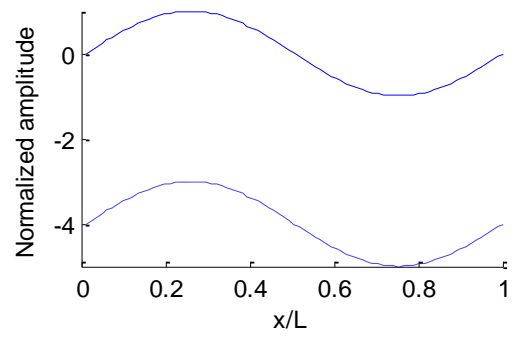

(c) Mode 3

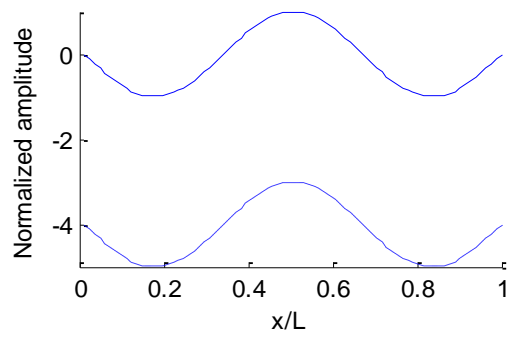

(e) Mode 5

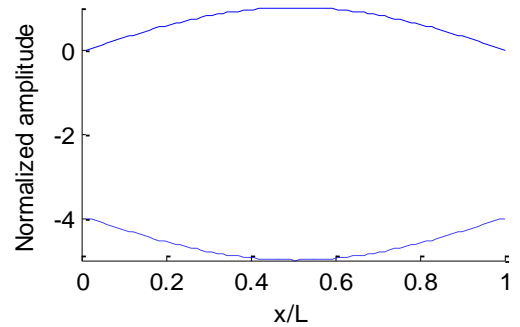

(b) Mode 2

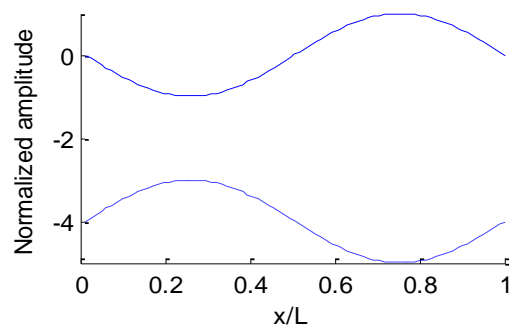

(d) Mode 4

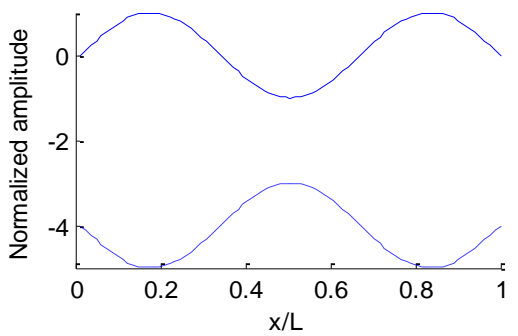

(f) Mode 6

Fig. 3. The first six mode shapes 
In Tab. 1 are listed the six lowest natural frequencies of the double-beam without a concentrated mass obtained by three methods. Fig. 3 shows the first six mode shapes of the double-beam obtained by the present work. As can be seen from Tab. 2 and Fig. 3, the first six natural frequencies and mode shapes of the present work are in close agreement with Ref. [1] and [2]. Especially, the natural frequencies obtained by the present method are in excellent agreement with Ref. [2].

Since there is no analogous model of a double beam carrying a concentrated mass as presented in the present study in the literature, in order to validate the present method the double beam is reduced to a single beam by removing the variables of the auxiliary beam and the elastic medium from Eqs. (1)-(14). The simulation results obtained by the present method are then compared to Ref. [12]. Tab. 2 presents the first five natural frequencies of the double cantilever beam carrying a concentrated mass at the tip by two methods. From this table, a close agreement between the results obtained from the present work and Ref. [12] can be observed.

Table 2. Natural frequencies of a single cantilever beam with a lumped mass at the tip

\begin{tabular}{|c|c|c|c|}
\hline \multirow{3}{*}{$\begin{array}{c}\text { Mass ratio } \\
\mu=\frac{m}{\rho A L}\end{array}$} & $\begin{array}{c}\text { Non-dimensional } \\
\text { natural frequency }\end{array}$ & Ref. [12] & Present paper \\
\hline \multirow{5}{*}{0} & $\bar{\omega}_{i}=\omega_{i} \sqrt{\frac{\rho A L^{4}}{E I}}$ & & \\
\cline { 2 - 4 } & $\bar{\omega}_{1}$ & 3.5159 & 3.5160 \\
\cline { 2 - 4 } & $\bar{\omega}_{2}$ & 22.0350 & 22.0345 \\
\cline { 2 - 4 } & $\bar{\omega}_{3}$ & 61.6960 & 61.6971 \\
\cline { 2 - 4 } & $\bar{\omega}_{4}$ & 122.9000 & 120.9013 \\
\hline \multirow{5}{*}{0.5} & $\bar{\omega}_{5}$ & 199.8600 & 199.8568 \\
\cline { 2 - 4 } & $\bar{\omega}_{1}$ & 2.0163 & 2.0288 \\
\cline { 2 - 4 } & $\bar{\omega}_{2}$ & 16.9010 & 17.0916 \\
\cline { 2 - 4 } & $\bar{\omega}_{3}$ & 51.7010 & 52.3210 \\
\cline { 2 - 4 } & $\bar{\omega}_{4}$ & 106.0500 & 107.3564 \\
\hline & $\bar{\omega}_{5}$ & 180.1300 & 182.3473 \\
\hline
\end{tabular}

\subsection{Influence of the concentrated mass on the free vibration of the intact double-beam}

In this section the MLFs of natural frequencies are established to study the influence of the position of concentrated mass on natural frequencies of the double-beam. The simulations for this case study showed that the concentrated mass only influences the natural frequency, while its influence on the mode shape is very small and cannot be seen visually. However, the trends of MLFs are related to the mode shapes. Thus, in order to establish relationship between the influence of the concentrated mass on the natural frequencies and the mode shapes, the corresponding mode shapes of the double 
beam without a concentrated mass are presented together with the MLFs as shown in Fig. 4. As can be seen from Figs. 4(a), 4(b), 4(c), 4(d), the first two natural frequencies decrease gradually as the concentrated mass is moved from the two ends to the middle of the double-beam where the amplitudes of the first two mode shapes are largest. Meanwhile, the third frequency decreases gradually from the fixed ends as the mass is moved to the positions of about $2.2 \mathrm{~m}$ and $7.8 \mathrm{~m}$ where the amplitude of the third mode shape is largest as illustrated in Figs. 4(e), 4(f). The third natural frequency increases when the mass is moved from these two positions to the middle of the beam where the amplitude of the third mode shape is zero which is known as the node of the mode shape.

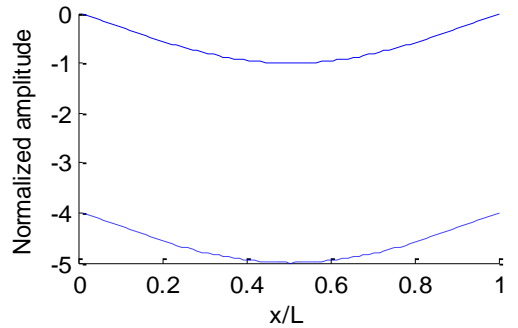

(a) The $1^{\text {st }}$ mode shape

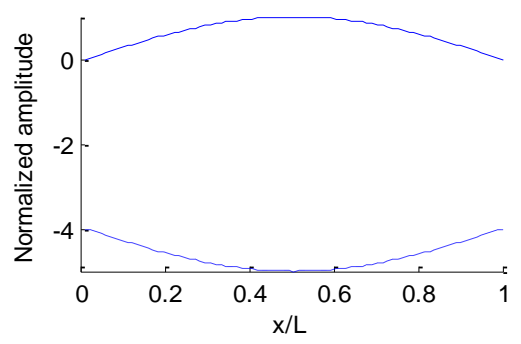

(c) The $2^{\text {nd }}$ mode shape

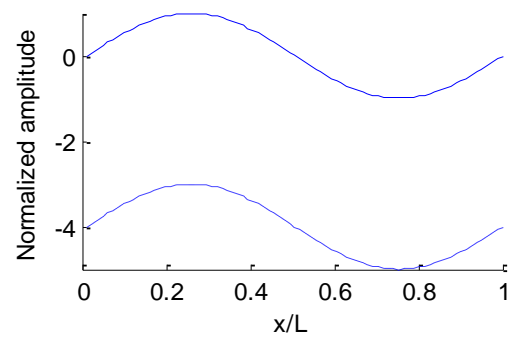

(e) The $3^{r d}$ mode shape

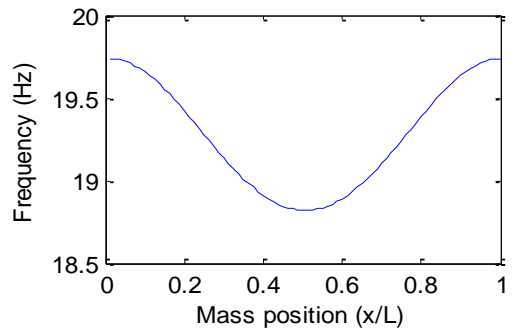

(b) MLF of the $1^{\text {st }}$ frequency

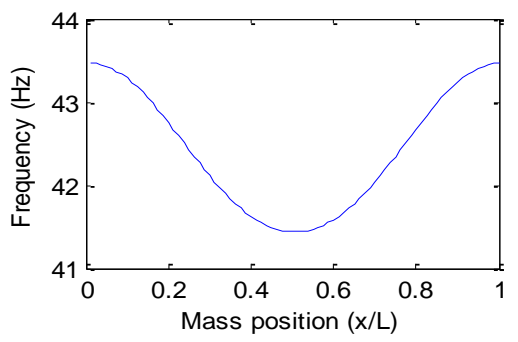

(d) MLF of the $2^{\text {nd }}$ frequency

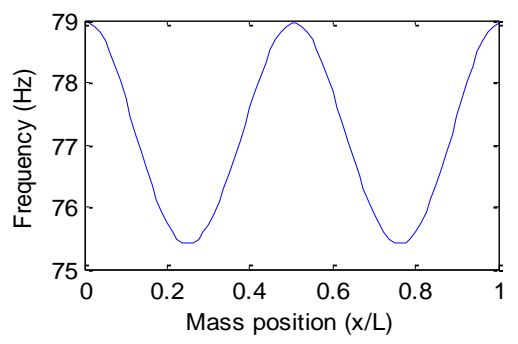

(f) MLF of the $3^{\text {rd }}$ frequency

Fig. 4. The first three mode shapes and MLFs

From these results, it is concluded that the influence of the concentrated mass is large when the mass is located at the large amplitude position of the mode shape and vice versa. The MLFs have local minima when the concentrated mass is located at the 
largest amplitude positions of the mode shapes. While, the MLFs have local maxima when the concentrated mass is located at the nodes of the mode shapes.

\subsection{Influence of the concentrated mass on the natural frequencies of the cracked double- beam and its application for crack detection using wavelet transform}

As discussed in the above section, the natural frequencies of the double-beam change when there is a concentrated mass. The changes in natural frequencies caused by the concentrated mass depend on the location of mass. Moreover, as can be found from the literature, when there is a crack, the natural frequencies of a beam change and the changes in natural frequencies depend on the position of the crack. Therefore, it is expected that when the concentrated mass and the crack are located close together there might be irregular changes in the natural frequencies. However, numerical simulations have shown that the irregular changes in the natural frequency caused by the crack and concentrated mass is small and difficult to be inspected visually. As an example, Fig. 5 presents the MLFs of the first three natural frequencies of the simply supported double beam having two cracks in the main beam with depths of $40 \%$ of the main beam height located at positions $3 \mathrm{~m}$ and $6.5 \mathrm{~m}$. Obviously, no irregular change can be observed visually from this figure.

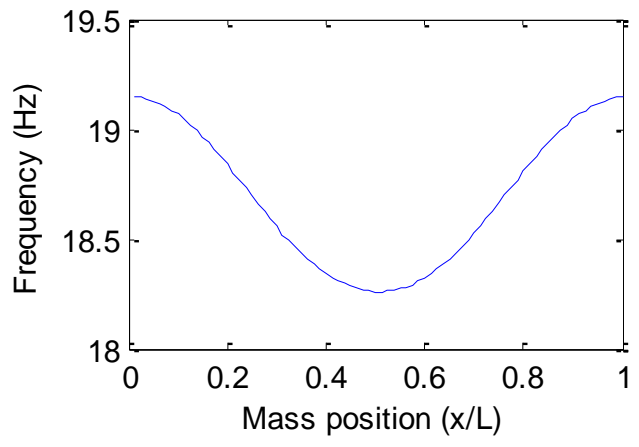

(a) MLF of the $1^{\text {st }}$ natural frequency

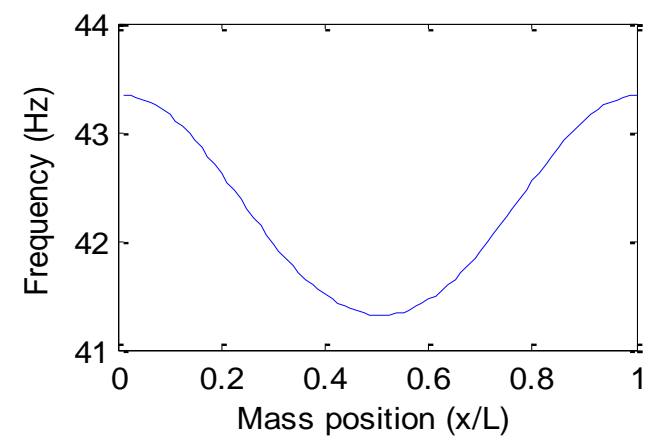

(b) MLF of the $2^{\text {nd }}$ natural frequency

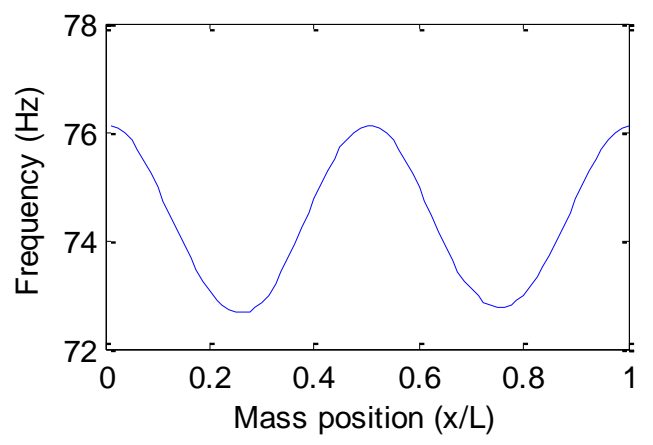

(c) MLF of the $3^{\text {rd }}$ natural frequency

Fig. 5. MLFs of the cracked double simply supported beam with the crack depth of $40 \%$ 
In order to reveal the irregular change in the MLFs at the crack positions, the difference $d f$ between the MLFs of the first frequency of the double beam with and without cracks are calculated by subtracting the MLF of the cracked double beam to the MLF of the intact double beam. Fig. 6 presents the difference $d f$ between the MLF of the intact double beam and the cracked double beam with different levels of the crack depth. As can be seen from this figure, there are two sharp changes in the graph of $d f$ when the crack depth ranges from $5 \%$ to $40 \%$. This result may be useful for crack detection: the crack location can be detected by the positions of the irregular changes in the graph of $d f$.

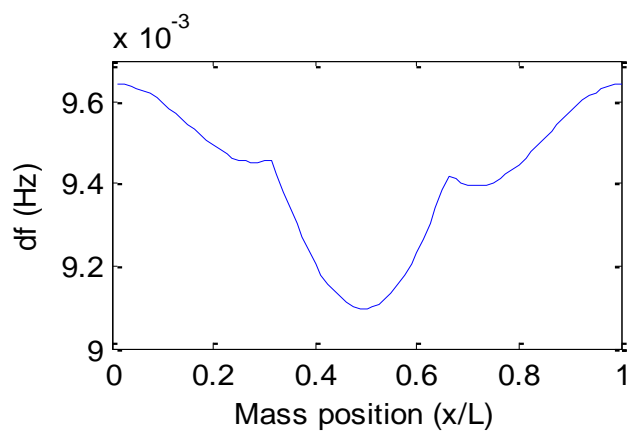

(a) Crack depth $5 \%$

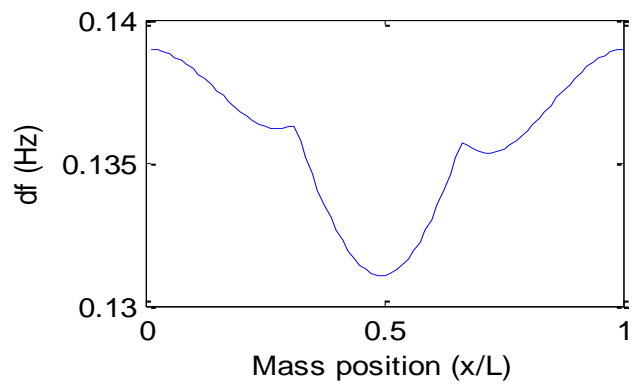

(c) Crack depth $20 \%$

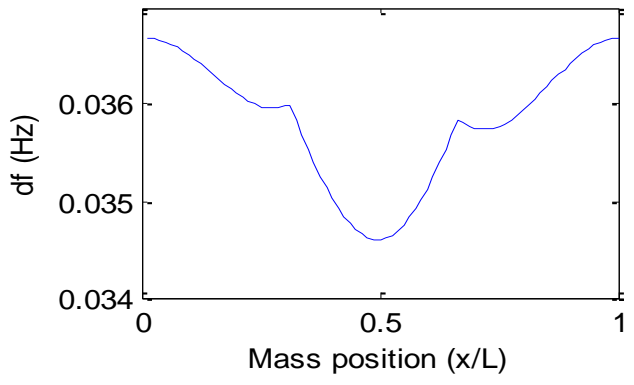

(b) Crack depth $10 \%$

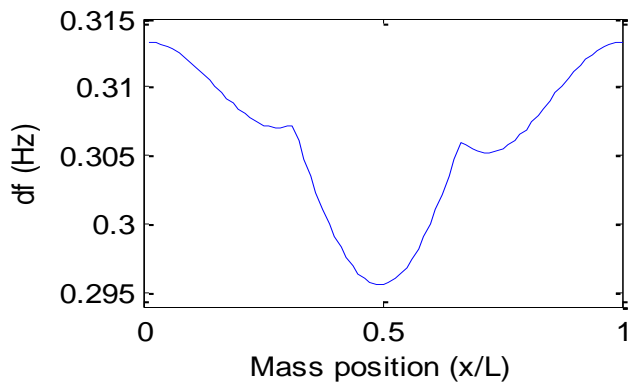

(d) Crack depth $30 \%$

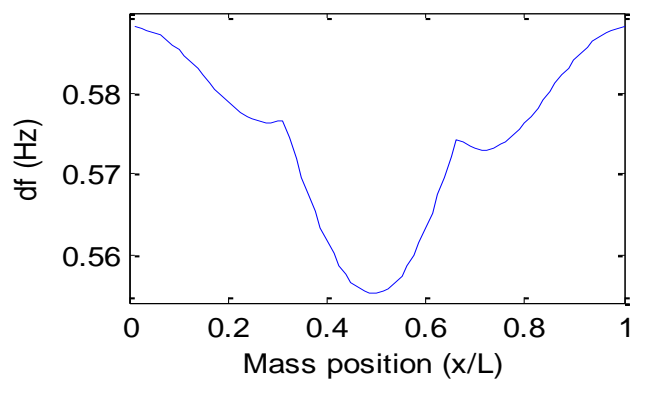

(e) Crack depth $40 \%$

Fig. 6. The difference $d f$ between MLFs of the cracked and intact double simply supported beams with different levels of the crack depth 
However, the MLF of an intact double beam as a baseline data is not always available in practice. Therefore, in order to inspect the irregular changes in the MLF caused by the concentrated mass and cracks, the wavelet transform can be used since the wavelet transform uses small wavelike functions which have local properties that are useful to analyse the hidden details or irregular changes contained in signals. In this study, the base line data is assumed to be unknown. Thus, the investigation of irregular changes in the MLFs using wavelet transform will be applied and presented as follows.

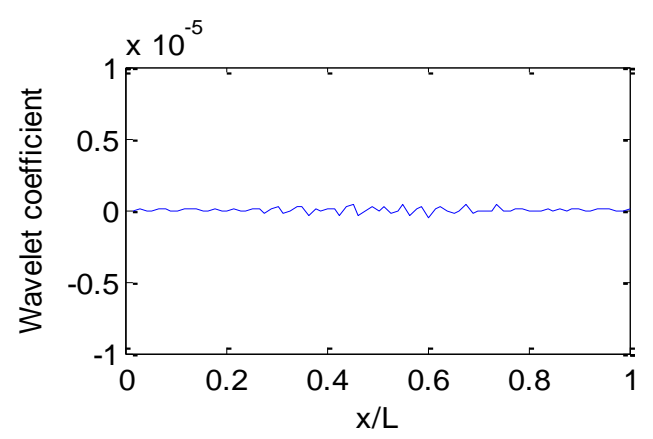

(a) Crack depth $0 \%$

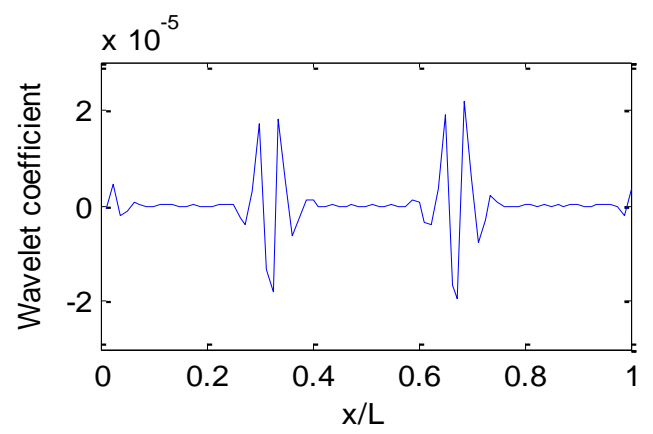

(c) Crack depth $10 \%$

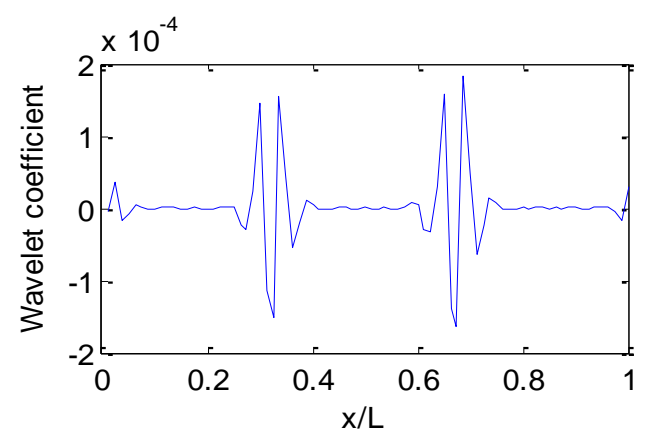

(e) Crack depth $30 \%$

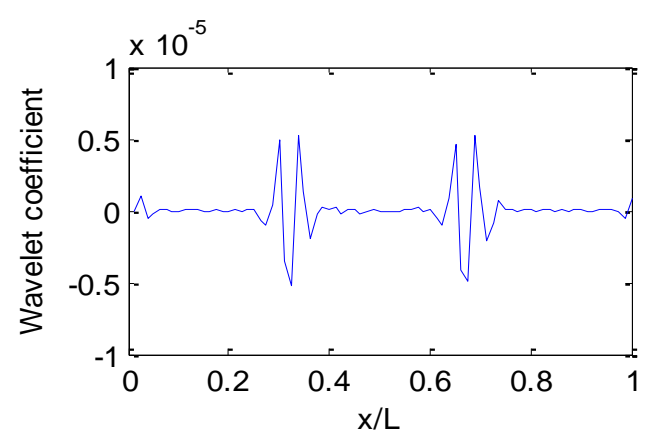

(b) Crack depth $5 \%$

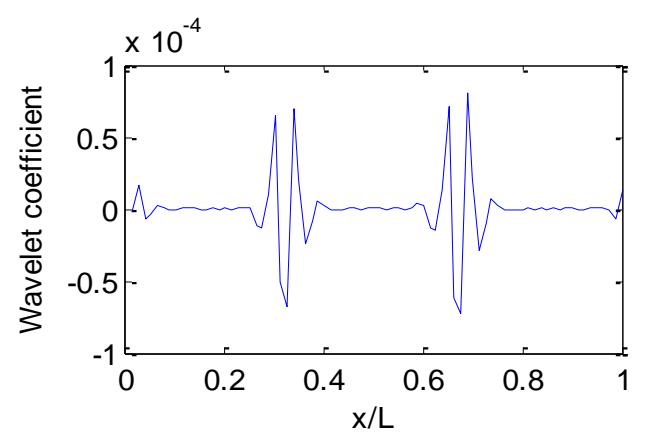

(d) Crack depth $20 \%$

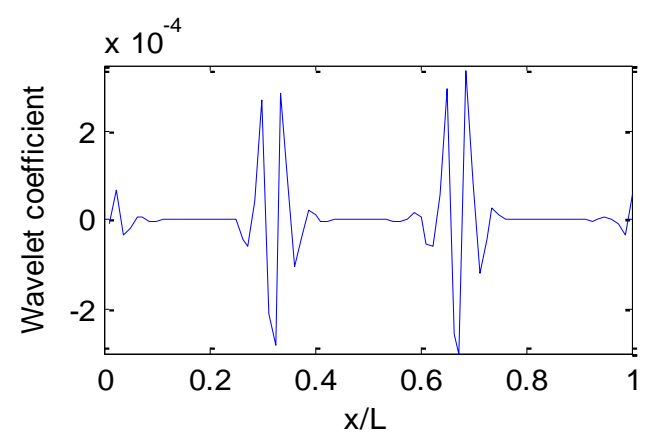

(f) Crack depth $40 \%$

Fig. 7. Wavelet transform of the MLF of the first natural frequency 
The wavelet transform is defined as follows [27]

$$
W(a, b)=\frac{1}{\sqrt{a}} \int_{-\infty}^{+\infty} f(t) \psi^{*}\left(\frac{t-b}{a}\right) d t,
$$

where $a$ is a real number called scale or dilation, $b$ is a real number called position, $W(a, b)$ are wavelet coefficients at scale $a$ and position $b, f(t)$ is input signal, $\psi\left(\frac{t-b}{a}\right)$ is wavelet function and $\psi^{*}\left(\frac{t-b}{a}\right)$ is complex conjugate of $\psi\left(\frac{t-b}{a}\right)$.

Let us apply the wavelet transform for the MLF of the first natural frequency of the double-beam having two cracks located arbitrary in the main beam. In this simulation, the two cracks with an identical depth are assumed to be located at $0.3 \mathrm{~m}$ and $0.65 \mathrm{~m}$. Six levels of the crack depth from $0 \%$ to $40 \%$ of the main beam height are applied.

Fig. 7 shows the wavelet transforms of the MLF of the first natural frequency with different crack depth levels. As can be seen from Fig. 7(a), when there is no crack there is no significant peak in the wavelet transform. However, when there are two small cracks with depth of $5 \%$, there are significant peaks in the wavelet transform at positions of 3 $\mathrm{m}$ and $6.5 \mathrm{~m}$ which are the locations of the cracks as presented in Fig. 7(b). When the crack depth increases from $10 \%$ to $40 \%$, the values of these peaks increase significantly as illustrated in Figs. 7(c), 7(d), 7(e), 7(f). These results imply that when there are cracks, the first frequency changes sharply when the concentrated mass is located at the positions of the cracks. This can be useful for crack detection: the crack locations can be detected by the locations of the significant peaks in the wavelet transform of the MLF of the first natural frequency.

\section{CONCLUSION}

In this paper, the influence of a concentrated mass on the natural frequencies of a double-beam consisting of two different simply supported beams is presented. When there is a concentrated mass the natural frequencies of the double-beam change. The changes in natural frequencies depend on the location of the mass and the mode shapes of the double-beam. These natural frequencies decrease gradually as the mass is moved from the nodes of the corresponding mode shapes at which the amplitude of the mode shapes are zero to the positions where the amplitudes of the corresponding mode shapes are largest.

The natural frequencies change sharply when the concentrated mass is located close to the crack positions. The sharp changes in natural frequencies can be detected by significant peaks in the wavelet transform of the MLFs. This can be useful for crack detection: the crack locations can be determined by the locations of the significant peaks in the wavelet transform of MLFs. However, the MLF of the first natural frequency can be applied efficiently for crack detection while the MLFs of higher natural frequencies are not recommended.

The proposed method for crack detection can be applied for detecting cracks with depths as small as $5 \%$. 


\section{ACKNOWLEDGEMENT}

This paper was sponsored by the Vietnam National Foundation for Science and Technology Development (NAFOSTED) 107.02-2014.01.

\section{REFERENCES}

[1] Z. Oniszczuk. Free transverse vibrations of elastically connected simply supported doublebeam complex system. Journal of Sound and Vibration, 232, (2), (2000), pp. 387-403. doi:10.1006/jsvi.1999.2744.

[2] Q. Mao. Free vibration analysis of elastically connected multiple-beams by using the Adomian modified decomposition method. Journal of Sound and Vibration, 331, (11), (2012), pp. 2532-2542. doi:10.1016/j.jsv.2012.01.028.

[3] Z. Oniszczuk. Forced transverse vibrations of an elastically connected complex simply supported double-beam system. Journal of Sound and Vibration, 264, (2), (2003), pp. 273-286. doi:10.1016/s0022-460x(02)01166-5.

[4] S. S. Rao. Natural vibrations of systems of elastically connected Timoshenko beams. The Journal of the Acoustical Society of America, 55, (6), (1974), pp. 1232-1237. doi:10.1121/1.1914690.

[5] Y.-H. Chen and J.-T. Sheu. Beam on viscoelastic foundation and layered beam. Journal of Engineering Mechanics, 121, (2), (1995), pp. 340-344. doi:10.1061/(asce)0733-9399(1995)121:2(340).

[6] M. Shamalta and A. V. Metrikine. Analytical study of the dynamic response of an embedded railway track to a moving load. Archive of Applied Mechanics, 73, (1-2), (2003), pp. 131-146. doi:10.1007/s00419-002-0248-3.

[7] H. V. Vu, A. M. Ordonez, and B. H. Karnopp. Vibration of a double-beam system. Journal of Sound and Vibration, 229, (4), (2000), pp. 807-822. doi:10.1006/jsvi.1999.2528.

[8] J. M. Seelig and W. H. Hoppmann. Impact on an elastically connected double-beam system. Journal of Applied Mechanics, 31, (4), (1964), pp. 621-626. doi:10.1115/1.3629723.

[9] H. Salarieh and M. Ghorashi. Free vibration of timoshenko beam with finite mass rigid tip load and flexural-torsional coupling. International Journal of Mechanical Sciences, 48, (7), (2006), pp. 763-779. doi:10.1016/j.ijmecsci.2006.01.008.

[10] W. H. Liu and F. H. Yeh. Free vibration of a restrained non-uniform beam with intermediate masses. Journal of Sound and Vibration, 117, (3), (1987), pp. 555-570. doi:10.1016/s0022460x(87)80074-3.

[11] J. S. Wu and T. L. Lin. Free vibration analysis of a uniform cantilever beam with point masses by an analytical-and-numerical-combined method. Journal of Sound and Vibration, 136, (2), (1990), pp. 201-213. doi:10.1016/0022-460x(90)90851-p.

[12] J. R. Banerjee. Free vibration of beams carrying spring-mass systems - A dynamic stiffness approach. Computers \& Structures, 104, (2012), pp. 21-26. doi:10.1016/j.compstruc.2012.02.020.

[13] W. H. Liu and C. C. Huang. Free vibration of restrained beam carrying concentrated masses. Journal of Sound and Vibration, 123, (1), (1988), pp. 31-42. doi:10.1016/s0022-460x(88)80075-0.

[14] R. P. Goel. Vibrations of a beam carrying a concentrated mass. Journal of Applied Mechanics, 40, (3), (1973), pp. 821-822. doi:10.1115/1.3423102.

[15] Y. S. Lee and M. J. Chung. A study on crack detection using eigenfrequency test data. Computers \& Structures, 77, (3), (2000), pp. 327-342. doi:10.1016/s0045-7949(99)00194-7.

[16] P. Gudmundson. Eigenfrequency changes of structures due to cracks, notches or other geometrical changes. Journal of the Mechanics and Physics of Solids, 30, (5), (1982), pp. 339-353. doi:10.1016/0022-5096(82)90004-7. 
[17] A. K. Pandey, M. Biswas, and M. M. Samman. Damage detection from changes in curvature mode shapes. Journal of Sound and Vibration, 145, (2), (1991), pp. 321-332. doi:10.1016/0022460x(91)90595-b.

[18] M. T. Vakil-Baghmisheh, M. Peimani, M. H. Sadeghi, and M. M. Ettefagh. Crack detection in beam-like structures using genetic algorithms. Applied Soft Computing, 8, (2), (2008), pp. 11501160. doi:10.1016/j.asoc.2007.10.003.

[19] S. Orhan. Analysis of free and forced vibration of a cracked cantilever beam. NDT \& E International, 40, (6), (2007), pp. 443-450. doi:10.1016/j.ndteint.2007.01.010.

[20] N. Khaji, M. Shafiei, and M. Jalalpour. Closed-form solutions for crack detection problem of Timoshenko beams with various boundary conditions. International Journal of Mechanical Sciences, 51, (9), (2009), pp. 667-681. doi:10.1016/j.ijmecsci.2009.07.004.

[21] S. Caddemi and I. Calio. Exact closed-form solution for the vibration modes of the EulerBernoulli beam with multiple open cracks. Journal of Sound and Vibration, 327, (3), (2009), pp. 473-489. doi:10.1016/j.jsv.2009.07.008.

[22] S. Zhong and S. O. Oyadiji. Detection of cracks in simply-supported beams by continuous wavelet transform of reconstructed modal data. Computers $\mathcal{E}$ Structures, 89, (1), (2011), pp. 127-148. doi:10.1016/j.compstruc.2010.08.008.

[23] K. V. Nguyen. Mode shapes analysis of a cracked beam and its application for crack detection. Journal of Sound and Vibration, 333, (3), (2014), pp. 848-872. doi:10.1016/j.jsv.2013.10.006.

[24] G. R. Liu and S. S. Quek. The finite element method: A practical course. Linacre House, Jordan Hill, Oxford, (2003).

[25] Y. H. Lin and M. W. Trethewey. Finite element analysis of elastic beams subjected to moving dynamic loads. Journal of Sound and Vibration, 136, (2), (1990), pp. 323-342. doi:10.1016/0022460x(90)90860-3.

[26] G. L. Qian, S. N. Gu, and J. S. Jiang. The dynamic behaviour and crack detection of a beam with a crack. Journal of Sound and Vibration, 138, (2), (1990), pp. 233-243. doi:10.1016/0022460x(90)90540-g.

[27] X. Q. Zhu and S. S. Law. Wavelet-based crack identification of bridge beam from operational deflection time history. International Journal of Solids and Structures, 43, (7), (2006), pp. 2299 2317. doi:10.1016/j.ijsolstr.2005.07.024. 